\title{
OPERAÇÕES DE RESPOSTA A DESASTRES: PROPOSTA DE UM MODELO DE GESTÃO E DE UM PROTÓTIPO DE BANCO DE DADOS
}

\section{DISASTER RESPONSE OPERATIONS: PROPOSAL OF A MANAGEMENT MODEL AND A DATABASE PROTOTYPE}

\author{
Maria Aparecida Garcia Bastos* E-mail:mariagarciabl@gmail.com \\ Renata Albergaria de Mello Bandeira* E-mail: re.albergaria@gmail.com \\ Vania Barcellos Gouvêa Campos* E-mail: vania@ime.eb.br \\ *Instituto Militar de Engenharia (IME), Rio de Janeiro, RJ
}

\begin{abstract}
Resumo: $O$ presente trabalho apresenta uma revisão das experiências de seis instituições internacionais e uma brasileira em operações de resposta a desastres naturais. Nesta revisão, procurou-se identificar as principais estratégias de atuação que possam vir a subsidiar o processo de tomada de decisão e ação nestas circunstancias. Com base nas análises realizadas, propõe-se um framework para subsidiar a organização das operações de apoio humanitário em desastres naturais no Brasil. A partir do conhecimento sintetizado neste framework, realiza-se a análise e estruturação de um banco de dados desenvolvido visando apoiar a operação de resposta a catástrofes, relacionando informações sobre aspectos como população, profissionais da saúde, transportes etc. Enfim, apresenta-se um protótipo desse banco de dados desenvolvido com o objetivo de amparar a tomada de decisões e execução de processos operacionais no pós-catástrofe, tendo como alvo principal de utilização as regiões de alta vulnerabilidade, que apresentam histórico de catástrofes.
\end{abstract}

Palavras-chave: Desastres naturais. Operações humanitárias. Gestão de desastres. Framework. Banco de dados.

Abstract: This paper reviews experiences of six international institutions and a Brazilian organization in disaster response operations, consolidating the main processes related to the operation and management of this type of humanitarian assistance. In this review, key strategic actions that may support the decision-making and operational processes in these circumstances were identified. Based on this analysis, we propose a framework for supporting the organization of relief response operations to natural disasters in Brazil. We also present the analysis and structure of a database designed to support this type of humanitarian operation, relating information on aspects such as population, health care, transport etc. Finally, a prototype of the database developed is presented, aiming to assist the decision-making process and organization of relief operation in post-disaster events.

Keywords: Natural disasters. Humanitarian operations. Disaster management. Framework. Database.

\section{INTRODUÇÃO}

Nas últimas três décadas, a ocorrência de desastres naturais aumentou significativamente, tendo a taxa de catástrofes se elevado de 50 para 400 por ano (Kovacs e Spens, 2009). Para os próximos 50 anos, é previsto que esta taxa aumente em até cinco vezes (Thomas e Kopczack, 2005). Entre 2002 e 2011, 270 milhões de vítimas foram atingidas por desastres naturais, que causaram o óbito de 
110 mil indivíduos (Guha-Sapir et al., 2013). No Brasil, entre 2000 e 2010, os desastres naturais causaram 1.696 óbitos, atingindo 7.543 .517 pessoas e gerando um custo de U\$ 1.547 milhões, sendo principalmente originados por inundações, enchentes, deslizamentos e tempestades (SEDEC, 2009).

Imediatamente após a ocorrência desses desastres, operações humanitárias são iniciadas com a intenção de prestar rápida assistência às vítimas em diferentes aspectos, tais como remoção ou resgate de /mortos e feridos, distribuição de recursos, fornecimento de alimentos, abrigo e assistência médica, bem como restaurar o acesso a locais remotos. Desta forma, há uma grande mobilização com o intuito de prover auxílio às vítimas. Entretanto, são vários grupos (organizações humanitárias locais e internacionais, organizações privadas, governo, militares e voluntários) com objetivos, perspectivas do problema e estruturas diferentes, agindo em um cenário onde há perda de serviços e infraestruturas essenciais, em um ambiente, algumas vezes, desprovido de segurança. Tais características, aliadas ao cenário instável e aleatório imposto por um desastre natural, aumentam a complexidade da gestão de operações humanitárias neste contexto.

Os eventos na Região Serrana do Rio de Janeiro, em 2011, expuseram o despreparo no Brasil para a condução deste tipo de operação humanitária, onde o tempo e a qualidade do atendimento são fatores significativos para a redução dos prejuízos sociais e econômicos decorrentes. Acredita-se que o planejamento de ações de resposta imediata e de recuperação é essencial para aumentar a eficiência das operações de resposta a desastres naturais no Brasil. Neste sentido, identificouse a necessidade de desenvolvimento de um estudo para determinar e mapear, com base na experiência de instituições internacionais, os principais processos relacionados à gestão da ajuda humanitária em desastres naturais, de maneira a subsidiar este tipo de operação no Brasil. Portanto, o objetivo deste trabalho é a síntese de conceitos logísticos aplicados às operações de resposta a desastres e sua avaliação a fim de contribuir com uma metodologia que possa auxiliar à atuação de instituições governamentais em atendimento as vítimas de desastres naturais no Brasil.

A metodologia de desenvolvimento deste trabalho compreendeu inicialmente a identificação das principais atividades e ações desenvolvidas pelos principais órgãos internacionais e nacionais de ajuda humanitária. A partir desta revisão, foram 
identificados os princípios básicos de atuação que possibilitou a elaboração de um modelo de ação (framework), que visa subsidiar a atuação de instituições no Brasil no caso de catástrofes. Uma primeira versão deste framework foi avaliada por um grupo de especialista cujas sugestões e críticas foram consideradas na versão final do mesmo. Ainda, com o objetivo de subsidiar as ações em locais onde ocorrem com frequência os desastres, foi proposto um banco de dados, cujas informações possibilitam agilizar todas as ações propostas no framework.

Assim sendo, o artigo apresenta uma revisão das experiências de seis instituições internacionais e uma brasileira em operações de resposta a desastres naturais (seção 2). Na seção 3, propõe-se um framework para subsidiar a organização das operações logísticas de apoio humanitário em desastres naturais no Brasil. Na seção 4, a partir do conhecimento sintetizado neste framework, o protótipo de um banco de dados desenvolvido com o objetivo de amparar a tomada de decisões e execução de processos no pós-catástrofe, tendo como alvo principal de utilização as regiões de alta vulnerabilidade, que apresentam histórico de catástrofes.

\section{MODELOS DE ATUAÇÃO DAS AGÊNCIAS INTERNACIONAIS E DO BRASIL}

Embora as características operacionais das ações de resposta possam variar de acordo com o tipo de desastre (Balcick et al., 2010), os princípios de gestão são semelhantes, especialmente para eventos que ocorrem repentina e intensamente (Gad-el-Hak, 2008). Para Gad-el-Hak (2008), o alcance do desastre é que determina a extensão da resposta requerida. Assim, pode-se definir um padrão de conduta para operações de resposta a desastres, criando-se um método estruturado em conhecimentos anteriores e na experiência de instituições internacionais de ajuda humanitária.

Neste sentido, foram analisados modelos de atuação apresentados em sete manuais de gestão e operação da ajuda em desastres. Dois destes trabalhos são de instituições voltadas para o estudo e disseminação de conceitos de logística no setor humanitário (Logistics Operational Guide da Log Cluster Org e Humanitarian Logistics: Enabling Disaster Response do Instituto Fritz), dois são manuais de atuação em operações de resposta a catástrofes (National Response Framework 
dos Estados Unidos e The Guide to the National Civil Defence Emergency Management Plan da Nova Zelândia), dois são manuais de organizações não governamentais com vasta experiência em logística humanitária (Emergency Field Handbook - A Guide For UNICEF Staff da UNICEF e Emergency Field Operations Pocketbook do WFP) e por último, o Manual Gerenciamento de Desastres - Sistema de Comando em Operações (Oliveira, 2009) utilizado pela Defesa Civil brasileira e que trata da criação e administração de um sistema de comando da operação de resposta a catástrofes no Brasil. Este não aborda de forma sistemática os processos de ajuda pós-catástrofe, mas foi considerado relevante ao estudo por ser uma publicação importante dentro da pouca produção nesta área no Brasil.

A seguir apresenta-se uma breve descrição das principais ações e propósitos dos manuais analisados:

- O Guia de Logística Operacional, (http://log.logcluster.org) é uma ferramenta desenvolvida a partir da compilação de vinte e oito manuais acadêmicos, de organizações não governamentais e privadas visando aumentar a eficácia e rapidez de resposta no atendimento das necessidades da ajuda humanitária. O Guia de Logística Operacional traz práticas a serem adotadas em operações de resposta a desastres, além de estabelecer objetivos gerais a serem seguidos, dentro dos seguintes pressupostos: Avaliação e Planejamento, Aquisição, Transporte, Alfândega, Cadeia de Frio, Armazenagem e Gerenciamento de Estoque, Gestão de Frotas, Distribuição, Monitoramento e Avaliação, Controle de Qualidade.

- O documento Humanitarian Logistics: Enabling Disaster Response (Thomas, 2003) apresenta as principais atividades de logística que devem compor uma operação de resposta a desastre. São elas: Avaliação, Gestão da Ajuda, Planejamento Operacional, Mobilização, Operações Internas, Coordenação entre Agências e Relatórios. Este manual é recomendado pelo Instituto Fritz, uma organização sem fins lucrativos que trabalha junto a governos, empresas e outras instituições de ajuda humanitária com o objetivo de apontar soluções e as melhores práticas para resposta a desastres, foi fundado em 2001.

- O Emergency Field Handbook - A Guide for Unicef Staff desenvolvido pela 
UNICEF em 2005, apresenta um guia institucional sobre a atuação na área de suprimentos e logística em operações emergenciais. Contém informações essenciais e propostas sobre os métodos de orçamentos de transporte e frete, recomendações sobre procedimentos alfandegários e uma visão geral do que precisa ser considerado para se armazenar e transportar suprimentos na situação de emergência. Além disto, $O$ Emergency Field Handbook - UNICEF, 2005 - traz uma série de conceitos, que estão diretamente ligados à logística necessária em operações de desastres naturais, tais como as considerações sobre a cadeia de frio, apresentadas no tópico saúde e nutrição deste, e outros relevantes como os tratados no tópico higiene e saneamento do mesmo.

- O Guide to the National Civil Defence - Emergency Management Plan, publicado em 2006 e revisado em 2009 na Nova Zelândia, tem por objetivo prover efetivo gerenciamento e reduzir o impacto dos desastres nas comunidades e população deste país, através de uma resposta planejada e coordenada com a participação de todos os níveis governamentais. Este guia apresenta como são gerenciadas as respostas a emergências na Nova Zelândia, tendo como foco o gerenciamento de informações de emergência e o processo de evacuação.

- O National Response Framework (NRF), guia de como os Estados Unidos gerencia operações de resposta a emergências, propõe estruturas de coordenação para alinhar os principais papéis e responsabilidades em todo o país, ligando todos os níveis de governo, organizações não governamentais e do setor privado. A intenção é definir autoridades específicas e as melhores práticas para gerenciar incidentes que vão desde grave, mas local, a ataques terroristas ou desastres naturais catastróficos de larga escala. Este guia se baseia no Sistema Nacional de Gerenciamento de Incidentes (NIMS). São definidos neste guia, os princípios fundamentais de resposta a incidentes, os participantes, suas funções e estruturas que norteiam as operações respostam a catástrofes nos Estados Unidos.

- O World Food Program reúne conceitos derivados de sua experiência de atuação em operações humanitárias emergenciais na publicação 
denominada Emergency Field Operations Pocketbook, destinada a ser um manual usado em campo. Em seu capítulo 9 (Logistics), apresenta um checklist de Avaliações de Capacidade Logística (LCAs) que destaca pontos importantes a serem observados referentes à armazenagem de suprimentos e transportes, bem como uma série de conceitos tratando desde a cooperação entre agências até preceitos de comunicação que devem ser observados mesmo em situações emergenciais. De acordo com o WFP (2002), a avaliação logística deve englobar a verificação da segurança local, das principais rotas de transporte e das operações de controle, transporte e armazenamento, em termos de: (a) situação e capacidade atuais; (b) o impacto do desastre; e (c) qualquer mudança esperada no futuro previsível.

- O Manual Gerenciamento de Desastres - Sistema de Comando em Operações trata das bases de implantação de um comando unificado para resposta a desastres no Brasil. Este manual discute as estratégias necessárias à implantação deste comando, para padronizar ações de resposta em desastres, que permita que seus usuários adotem uma estrutura organizacional integrada para enfrentar as demandas e complexidades dos desastres, porém, diferentemente dos demais manuais analisados não foca no processo da ajuda humanitária. O Sistema de Comando em Operações funciona como uma ferramenta gerencial para planejar, organizar, dirigir e controlar os efeitos dos desastres, independentemente de sua causa, tamanho, configuração, localização ou complexidade.

Dentre as diretrizes propostas pelos guias analisados, ressalta-se a coincidência de alguns processos sugeridos, o que vem enfatizar a importância de sua aplicação em operações de resposta a desastres. Também cabe ressaltar que alguns processos são sugeridos por apenas uma das organizações, mas complementam o processo de resposta a desastres. Da análise realizada, foi possível caracterizar nove processos básicos adotados por estas agências:

- Mobilização, citada na maioria dos documentos avaliados, entretanto de maneira bastante sucinta como, por exemplo, no National Response Framework (FEMA, 2008), que sugere a necessidade de "ativar centros de 
operações e especialistas no assunto". O Manual Gerenciamento de Desastres - Sistema de Comando em Operações (Oliveira, 2009), que trata mais especificamente do processo de gestão da crise, detalha a mobilização e ressalta sua importância;

- Avaliação e o planejamento da operação, citados em todos os documentos como sendo primordial no pós-catástrofe. O processo de avaliação é tratado de forma mais detalhada pelo Logistics Operational Guide (http://log.logcluster.org) e complementado pelo Emergency Field Operations Pocketbook (WFP, 2002). Estes ressaltam detalhes como a necessidade de uma "avaliação situacional" para se identificar a extensão e gravidade da crise e de uma "avaliação logística" que visa à identificação das possibilidades e das necessidades mais prementes para se implantar processos satisfatórios na operação de resposta. As organizações sugerem o início da operação humanitária a partir do processo de avaliação. O planejamento da operação é detalhado no National Response Framework (FEMA, 2008), sob o título "Planejamento, um elemento crítico da resposta eficaz";

- Aquisição, apesar de também ser abordada pelo Humanitarian Logistics: Enabling Disaster Response (Thomas, 2003), tem sua melhor apresentação no texto do Logistics Operational Guide (http://log.logcluster.org) que define o processo de aquisição em quatro passos: (i) identificação das necessidades; (ii) especificação dos produtos e serviços; (iii) obtenção das fontes, definição e encomendas; e (iv) gestão de fornecedor para garantir a entrega correta no tempo certo. Essas duas organizações sugerem a correta especificação das necessidades como ponto de partida do processo de aquisição.

- Armazenagem e a gestão do estoque: são tratadas principalmente nos documentos Logistics Operational Guide (http://log.logcluster.org), Humanitarian Logistics: Enabling Disaster Response (Thomas, 2003), Emergency Field Handbook - A Guide For Unicef Staff (UNICEF, 2005) e Emergency Field Operations Pocketbook (WFP, 2002), sendo apresentadas essas informações sob os diferentes títulos de "Gestão da Ajuda", e "Instalações de Armazenamento" sendo entretanto complementar 
entre os documentos citados;

- Distribuição, abordada da melhor forma e em mais detalhes pelo Logistics Operational Guide (http://log.logcluster.org), sendo analisada a partir de três perspectivas: a circulação de mercadorias do ponto de compra ao ponto de uso, a circulação de mercadorias de um local para outro dentro da organização e a entrega ao usuário final;

- Transporte, tratado na maioria dos textos. Observa-se a importância de se investigar as rotas disponíveis, internas e de acesso à região afetada, bem como terminais de uso provável, os recursos da frota local do governo, particulares ou de instituições, assim como os possíveis gargalos e a possibilidade de superá-los. O documento Emergency Field Operations Pocketbook (WFP, 2002) sugere um completo checklist de informações necessárias para este processo a serem levantadas na avaliação da crise e consideradas no planejamento da operação;

- Controle de qualidade e monitoramento da operação, recomendado pelo Logistics Operational Guide (http://log.logcluster.org), pelo Humanitarian Logistics: Enabling Disaster Response (Thomas, 2003) e Emergency Field Handbook - A Guide For Unicef Staff (UNICEF, 2005), ressalta a necessidade do desenvolvimento de padrões de procedimentos e controle de qualidade dos fornecimentos. Os documentos analisados enfatizam que a logística nas cadeias humanitárias, por ser implantada em caráter emergencial, necessita de monitoramento contínuo para que falhas sejam corrigidas. O Logistics Operational Guide (http://log.logcluster.org) sugere aspectos a serem monitorados na operação de resposta e procedimentos de controle de qualidade nas emergências, sendo a melhor referência a ser considerada para este tópico, juntamente com o texto da Emergency Field Handbook - A Guide For Unicef Staff (UNICEF, 2005) que indica pontos a serem observados na garantia da qualidade e documentos a serem implantados;

- Coordenação entre agências, sugerida pelo Humanitarian Logistics: Enabling Disaster Response (Thomas, 2003) e fortemente aconselhado pelo National Response Framework (FEMA, 2008). A importância da coordenação entre agências é devida, principalmente, para evitar gastos 
com duplicação de processos, concorrência por transportes, armazenagem e pessoal, bem como facilitar o compartilhamento de informações visando desenvolver um processo operacional comum e sincronizar suas operações de resposta e recursos;

- Gestão e fluxo da informação, citada em todos os documentos analisados, estão descritos de forma mais completa no documento The Guide to the National Civil Defence Emergency Management Plan (Ministry of Civil Defence \& Emergency Management of New Zealand, 2009), sendo ele a melhor referência para este tópico e subsídio para aplicação prática.

Para uma visão geral da análise, relaciona-se, no Quadro 1, os processos básicos identificados e os documentos onde foram tratados. Os documentos estão identificados da seguinte forma: Logistics Operational Guide como Log Cluster, Humanitarian Logistics: Enabling Disaster Response como Inst. Fritz, Emergency Field Handbook como UNICEF, The Guide to the National Civil Defence - Emergency Management Plan como N.Z., o National Response Framework como FEMA e o Emergency Field Operations Pocketbook como WFP e o Manual Gerenciamento de Desastres - Sistema de Comando em Operações como SCO.

Quadro 1 - Principais Processos dos documentos analisados

\begin{tabular}{|c|c|c|c|c|c|c|c|}
\hline & Log & Inst. & UNICEF & NZ. & FEMA & WFP & SCO \\
\hline Mobilização & $\mathrm{X}$ & $\mathrm{X}$ & $\mathrm{X}$ & $\mathrm{X}$ & $\mathrm{X}$ & & $\mathrm{X}$ \\
\hline Avaliação e Planejamento & $\mathrm{X}$ & $\mathrm{X}$ & $\mathrm{X}$ & $\mathrm{X}$ & $\mathrm{X}$ & $\mathrm{X}$ & \\
\hline Aquisição & $\mathrm{X}$ & $\mathrm{X}$ & $\mathrm{X}$ & & $\mathrm{X}$ & & \\
\hline Arm. e gerencia de estoques & $\mathrm{X}$ & $\mathrm{X}$ & $\mathrm{X}$ & & & & \\
\hline Distribuição & $\mathrm{X}$ & $\mathrm{X}$ & $\mathrm{X}$ & & & & \\
\hline Transportes & $\mathrm{X}$ & $\mathrm{X}$ & $\mathrm{X}$ & & & & \\
\hline Gestão de Frotas & $\mathrm{X}$ & & & & & & \\
\hline Alfândega & $\mathrm{X}$ & $\mathrm{X}$ & $\mathrm{X}$ & & & $\mathrm{X}$ & \\
\hline Cadeia do Frio & $\mathrm{X}$ & & & & & & \\
\hline Evacuação & & & & $\mathrm{X}$ & & & \\
\hline Cont. de Qualidade $\mathrm{X}$ & $\mathrm{X}$ & $\mathrm{X}$ & $\mathrm{X}$ & & & & \\
\hline Coord. entre Agências & $\mathrm{X}$ & $\mathrm{X}$ & $\mathrm{X}$ & & $\mathrm{X}$ & & \\
\hline Gestão da Informação & $\mathrm{X}$ & $\mathrm{X}$ & $\mathrm{X}$ & $\mathrm{X}$ & $\mathrm{X}$ & $\mathrm{X}$ & \\
\hline Desmobilização & & & & & $\mathrm{X}$ & & \\
\hline
\end{tabular}

Entretanto, na prática, estes processos logísticos têm que ser operacionalizados em ações e atividades. Constata-se, no estudo dos manuais apresentados, a existência de um padrão de ações essenciais a serem 
desempenhadas em uma operação de resposta, que visam à garantia da sobrevivência dos vulneráveis e à minimização dos prejuízos materiais e sociais. Essas ações como, por exemplo, resgate e salvamento, distribuição de suprimentos básicos à sobrevivência, dependem intrinsecamente dos processos logísticos, o que direciona a uma ideia de um sistema de atuação no pós-catástrofe, onde podem se relacionar os processos logísticos essenciais para sucesso de cada ação. Na seção 3, desenvolve-se esse tema com base nas análises realizadas, sugerindo um framework a ser utilizado nas operações de resposta às catástrofes. O modelo proposto inclui as principais ações a serem realizadas na operação de resposta, detalhando as atividades envolvidas em cada uma destas ações.

\section{PROPOSTA DE FRAMEWORK: AÇÕES DE RESPOSTA IMEDIATA}

Como resultado das análises realizadas, propõe-se, nesta seção, um framework que compreende uma síntese das principais ações e atividades logísticas a serem aplicadas em operações de resposta a desastres naturais, conforme apresentado no Quadro 2. Para estruturação deste modelo, as ações e atividades foram grupadas em quatro etapas de acordo com uma ordem temporal de prioridades, que visa estabelecer uma organização do trabalho. Contudo, na prática, essas etapas podem naturalmente se sobrepor.

Pela análise realizada, verificou-se que o conjunto de ações apresentado é fundamental para o sucesso de uma operação de resposta a catástrofe, sendo que grande parte dessas ações é tratada no documento National Response Framework (FEMA, 2008) em seu capítulo 2, denominado "Ações de Resposta". O conjunto é complementado por ações sugeridas pelo The Guide to the National Civil Defence Emergency Management Plan (Ministry of Civil Defence \& Emergency Management of New Zealand, 2009), Emergency Field Handbook - A Guide For Unicef Staff (UNICEF, 2005), Logistics Operational Guide como Log Clus Logistics Operational Guide (http://log.logcluster.org), Humanitarian Logistics: Enabling Disaster Response (Thomas, 2003) e Emergency Field Operations Pocketbook (WFP, 2002) que realçam a necessidade das ações de Mobilização, Avaliação Situacional, Avaliação Logística, Gerenciamento da Ajuda e Restabelecimento da Normalidade.

Como parte da metodologia de desenvolvimento deste trabalho, uma versão 
preliminar do framework foi submetida à avaliação de dez profissionais ligados à área, entre eles oito pesquisadores de logística humanitária e dois com prática em operações de resposta a catástrofes. Um formulário com a primeira versão do framework e algumas perguntas foi enviado a esses profissionais. As correções indicadas e outras sugestões foram incorporadas ao framework proposto no Quadro 2. Este quadro apresenta a estrutura básica do framework com as principais ações e atividades a serem aplicadas em operações de resposta a desastres naturais.

Para melhor compreensão do framework apresentado, é necessário esclarecer os conceitos de etapa, ação e atividades. Para tal, é realizado um paralelo com alguns conceitos de gestão de projetos. Segundo o PMBOK Guide (2010), o ciclo de vida de um projeto consiste nas fases do mesmo que geralmente são sequenciais e às vezes se sobrepõem. Da mesma forma que as fases na Gestão de Projetos, as etapas definidas pelo framework visam segmentar a operação de resposta em subconjuntos lógicos para facilitar o gerenciamento. Logo, as etapas Reconhecimento, Salvamento, Manutenção e Desmobilização podem ser tratadas como fases de um projeto do tipo "operação de resposta", e assim se pode definir o ciclo de vida da operação de resposta.

Sendo assim, ações e atividades foram grupadas nestas quatro etapas (Reconhecimento, Salvamento, Manutenção e Desmobilização) de acordo com uma ordem temporal de prioridades, que visa estabelecer uma organização do trabalho. No entanto, na prática, essas etapas podem ser sobrepor. Paralelamente, as ações são consideradas como subfases, englobadas em cada etapa (fase), e seguem a lógica de atuação dentro daquela etapa, de forma que o conjunto destas ações visa atingir o objetivo geral da etapa. Neste paralelo, tem-se, por exemplo, como subfases da etapa Reconhecimento, as ações de Mobilização, Avaliação Situacional e Avaliação Logística, que cumprindo seus objetivos parciais, cumprem o objetivo geral da etapa (fase). 
Quadro 2 - Ações e atividades logísticas nas operações de resposta a desastres naturais

\begin{tabular}{|c|c|c|}
\hline Etapas & Ações em Campo & Atividades \\
\hline \multirow{6}{*}{ Reconhecimento } & Mobilização & Estabelecer centro de gerenciamento da crise \\
\hline & \multirow{2}{*}{ Avaliação Situacional } & Identificar a abrangência da crise \\
\hline & & Identificar a gravidade da crise \\
\hline & \multirow{3}{*}{ Avaliação Logística } & Identificar os recursos disponíveis na região atingida \\
\hline & & Identificar os recursos necessários à região atingida \\
\hline & & Definir base para operação dentro da região atingida \\
\hline \multirow{11}{*}{ Salvamento } & \multirow{3}{*}{$\begin{array}{l}\text { Primeiros } \\
\text { população }\end{array}$} & Localizar e resgatar os feridos \\
\hline & & Realizar triagem de atendimento \\
\hline & & Realizar atendimento \\
\hline & \multirow{5}{*}{$\begin{array}{l}\text { Proteção à população da área } \\
\text { atingida }\end{array}$} & Identificar as áreas de risco \\
\hline & & Identificar necessidades de evacuação ou proteção \\
\hline & & Identificar probabilidade de contaminação e doenças \\
\hline & & Divulgar informações sobre riscos e como evita-los \\
\hline & & Identificar as possibilidades de agravamento da crise \\
\hline & \multirow{3}{*}{$\begin{array}{l}\text { Garantia da sobrevivência e } \\
\text { restabelecimento das } \\
\text { condições essenciais }\end{array}$} & Fornecer água, suprimentos e abrigos \\
\hline & & Restabelecer rotas de acesso \\
\hline & & Rest. serviços de água, energia elétrica e comunicações \\
\hline \multirow{3}{*}{ Manutenção } & \multirow{3}{*}{ Gerenciamento da ajuda } & Gerenciar suprimentos e necessidades \\
\hline & & Gerenciar abrigos \\
\hline & & Avaliar e Monitorar os processos estabelecidos \\
\hline \multirow{2}{*}{ Desmobilização } & \multirow{2}{*}{$\begin{array}{l}\text { Restabelecimento da } \\
\text { normalidade }\end{array}$} & Recuperar a funcionalidade da região atingida \\
\hline & & Desmobilizar \\
\hline
\end{tabular}

Fonte: Org. dos Autores (2014)

A seguir apresenta-se um resumo das ações relacionadas no framework:

- Mobilização: compreende o estabelecimento do centro de gerenciamento da crise para gerir pessoal e estabelecer, juntamente à comunidade atingida, as ações essenciais na operação de resposta e o monitoramento e controle da atividade, tornando possível o aprendizado institucional. A coordenação desta ação deve ser responsabilidade da Defesa Civil, que precisa contar com a participação das prefeituras da região na forma das secretarias adequadas, ONGs, Forças Armadas e Corpo de Bombeiros. A partir de estabelecido o Centro de Gerenciamento da Crise, este passa a coordenar todas as demais ações. Uma decisão importante envolve a 
localização do Centro de Gerenciamento da Crise (CGC), que deve ser fora da zona de risco, considerando-se as possibilidades de agravamento da crise, entretanto deve estar o mais próximo possível da região impactada, e contar com rotas e trânsito livre até a base de operações localizada dentro da zona em crise. É importante que o local conte também com infraestrutura de comunicações ou que esta possa ser facilmente implantada. Os profissionais atuantes no CGC devem ter experiência em gerenciamento de crise e formação multidisciplinar, sendo extremamente importante que fiquem bem claras as atribuições e responsabilidades de cada um deles, evitando-se conflitos;

- Avaliação Situacional: o processo onde deverão ser destacadas informações como área atingida, população impactada, gravidade e extensão do desastre, etc. Como o alcance do desastre determina a extensão da resposta requerida (Gad-el-Hak, 2008), a avaliação situacional é essencial para o correto dimensionamento das demais ações e para o planejamento operacional. Na identificação da abrangência da crise, o pedido de informações sobre a crise deve ser divulgado em todos os meios disponíveis, além de conectar órgãos institucionais e ONGs na provável região atingida em busca de informações (informações telefônicas, internet, fotos de satélite, etc.). A região atingida deve ser mapeada através do cruzamento e avaliação das informações obtidas, atualizando constantemente conforme a obtenção de novos dados. Ainda, para definir a gravidade da crise, é importante definir os pontos prioritários de atendimento na região atingida, as necessidades de ajuda externa, bem como monitorar atividades em realização e redefinir gravidade e pontos prioritários de acordo com as informações recebidas;

- Avaliação Logística: compreende identificar rotas possíveis de acesso e internas a região atingida, terminais e suas capacidades, capacidade da frota local e do governo, possíveis armazéns ou abrigos, etc., possibilita a especificação das necessidades prementes à região em crise e a consequente gestão eficiente destas necessidades. Assim, além de de identificar os recursos disponíveis e determinar os necessários à região atingida, também se deve, nesta ação, definir a base para a operação 
dentro da região afetada pelo desastre. O documento da Cruz Vermelha, Guidelines for assessment in emergencies (2008), trata detalhadamente dos processos de levantamento de informações no pós-catástrofe, possibilitando um maior entendimento de como os processos de avaliação situacional e avaliação logística podem ser implementados;

- Primeiros socorros à população: O correto planejamento dos primeiros socorros a ser prestado à população atingida é fundamental para se minimizar os danos à saúde dos vulneráveis. Assim, deve-se localizar e resgatar os feridos, recolher os cadáveres, realizar a triagem de atendimento e, finalmente, realizar o atendimento em si. Convém destacar que o Manual de Medicina de Desastres - Volume I (Castro e Calheiros, 2007), da Secretaria Nacional de Defesa Civil - Ministério da Integração Nacional trata detalhadamente dos processos de resgate e atendimento médico no pós-catástrofe, incluindo a quantificação de pessoal e equipamentos;

- Proteção à população da área atingida: os riscos associados a desastres, principalmente nos assentamentos precários dos grandes centros urbanos, que ocupam áreas de riscos geotécnicos, em más condições de habitabilidade precisam ser avaliados de forma a minimizar as perdas e danos à sociedade decorrentes do agravamento da crise. Nesta ação, é fundamental identificar as áreas de risco, identificar as necessidades de evacuação ou proteção, identificar possibilidades de agravamento da crise e as possibilidades de contaminação e propagação de doenças, devendose divulgar informações sobre riscos e como proceder para evitá-los;

- Garantia da sobrevivência e restabelecimento das condições essenciais: a capacidade de empreender ações rápidas para garantir o funcionamento eficiente das instalações vitais, como hospitais e abrigos de emergência, o fornecimento ininterrupto de energia elétrica, rotas de acesso, abastecimento de água potável e saneamento é fundamental para se minimizar os prejuízos sociais e materiais da população atingida em uma catástrofe. Esta ação engloba o fornecimento de água, suprimentos e abrigo, o reestabelecimento de rotas de acesso e do fornecimento de água, 
energia elétrica e comunicações;

- Gerenciamento de ajuda: o planejamento das aquisições e entrega de ajuda humanitária para as vítimas requer um processo eficiente e estruturado, seguindo as seguintes atividades: (i) gerenciar suprimentos e necessidade, que abrange a distribuição organizada da ajuda e o gerenciamento de armazéns; (ii) gerenciar abrigos; e (iii) avaliar e monitorar os processos estabelecidos, que compreende a documentação da operação, o desenvolvimento de relatórios, a verificação do desempenho dos processos e a correção necessária destes. Até que se restabeleça a normalidade, este processo deve ser gerenciado com a participação de profissionais com conhecimentos e habilidades multidisciplinares por se tratar ainda de um processo logístico realizado em condições peculiares;

- Restabelecimento da normalidade: conforme os problemas vão sendo sanados e a situação estabilizada, diminuem os riscos e a complexidade, de modo que as tarefas necessárias vão exigindo cada vez menos articulação. É o momento de iniciar a desmobilização dos recursos empregados. Esse processo é gradual e contínuo, sendo necessária a recuperação da funcionalidade da região atingida. Nesta etapa, é importante prestar ajuda administrativa as vítimas (emissão de documentos etc), reconstruir a região atingida, com o reestabelecimento das condições de funcionamento das escolas, hospitais e pontos de atendimento médico de âmbito municipal, bem como encaminhar os que ainda permanecem nos abrigos às regiões estabelecidas para retorno. A desmobilização deve ser planejada e executada cuidadosamente para evitar o descontrole e a perda de equipamentos e materiais. Deve-se destinar à caridade as sobras de suprimentos e armazenar o material que possa ser reaproveitado (tendas, kits de desinfecção de água, etc.) junto à Defesa Civil da região.

Conforme a descrição apresentada, o framework proposto foi desenvolvido evidenciando-se ações e atividades consideradas essenciais ao gerenciamento da ajuda humanitária, de acordo com a importância percebida nas pesquisas. Tendo em vista a inexistência no Brasil de uma estrutura de operação imediata após desastres, este framework pode se tornar um instrumento bastante útil no atendimento à população, quando utilizado imediatamente a ocorrência do desastre pelas 
instituições governamentais responsáveis pelo gerenciamento e apoio a situação.

Conforme já mencionado, as ações foram agrupadas em etapas que definem uma ordem temporal de prioridades que visa auxiliar uma escalada na implantação da operação de resposta. Entretanto, é importante ressaltar que existem situações onde esta ordem pode ser revogada em benefício da operação, desde que ações mais urgentes não sofram prejuízos decorrentes da implantação antecipada de outras. A relação de atividades constantes no framework para cada ação não visa esgotar o contexto, mas apenas arrolar atividades primordiais nestas ações.

Além disto, é importante ressaltar que parte das atividades relacionadas às ações de Mobilização, Avaliação Situacional e Avaliação Logística pode ser previamente definida, cabendo apenas sua implementação após a ocorrência do desastre. Assim, como forma de atender a questão de preparo prévio e facilitar a operação de logística humanitária, propõe-se na seção 4 deste estudo, um modelo de banco de dados, elaborado a partir da consideração de todas as ações a serem realizadas na operação de resposta a catástrofes. Este modelo visa suprir as informações necessárias para o desempenho destas ações e que podem ser previamente disponibilizadas.

\section{ESTRUTURAÇÃo DE DADOS NECESSÁRIOS AOS PROCESSOS LOGÍSTICOS}

Para dar suporte à atuação segundo o framework proposto, foi desenvolvido um Modelo Conceitual de Banco de Dados visando catalogar informações prévias sobre a população da região, profissionais da saúde, transportes etc. Este banco de dados tem como objetivo amparar a tomada de decisões e execução de processos no pós-catástrofe em regiões de alta vulnerabilidade, que apresentam histórico de catástrofes, podendo ter ampla utilização. O custo de manutenção da base de dados sugerida é extremamente reduzido, sendo uma aplicação simples, mas de grande utilidade. Não foram encontradas aplicações similares no âmbito da logística humanitária no Brasil.

As ações de Mobilização, Avaliação Situacional e Avaliação Logística podem se beneficiar diretamente do cadastro prévio de informações, sendo estruturado o Modelo Conceitual do Banco de Dados de forma a suprir todas as informações necessárias a essas ações. Entretanto, outras ações operacionais, que dependem 
de dados atuais da catástrofe, ainda poderão se utilizar de informações levantadas para estas ações, como por exemplo, no atendimento aos feridos, as condições e localizações de hospitais. Apresenta-se o Modelo Conceitual do Banco de Dados e um protótipo desenvolvido nas seções 4.1 e 4.2 .

\subsection{Modelo conceitual do banco de dados}

O processo de Mobilização trata na prática do estabelecimento de um Centro de Gerencia mento da Crise e da definição de responsabilidades neste centro, bem como da mobilização de recursos e profissionais nas diversas outras ações. Para subsídio deste processo sugerem-se quatro entidades que não se relacionam entre si, a saber:

- Localização_centro: relaciona possíveis localizações do Centro de Gerenciamento da Crise; áreas, infraestrutura geral, segurança, responsável e contato.

- Responsabilidades_centro: relaciona os possíveis comandos de cada uma das áreas estabelecidas no SCO para o centro de gerenciamento da crise;

- Profissionais: relaciona os profissionais disponíveis para atuação na crise;

- Ongs/Instituições: relaciona as ONGs e instituições que podem fornecer apoio.

O processo de Avaliação Situacional consiste na prática do levantamento de dados relacionados à gravidade e abrangência da crise, que não podem ser previstas com exatidão, mas sim subsidiadas por informações sobre a região vulnerável. Esse processo apresenta como entidades a área e a população, relacionadas entre si:

- Área: relaciona cada região dentro da área de vulnerabilidade, sendo definida, entre outros, pelos atributos localização, tipo de vulnerabilidade a que está sujeita (enchente ou deslizamento), o grau de risco (determinado pela defesa civil);

- População: relaciona a localização, a população total da área e ainda atributos como a população de idosos, de portadores de necessidades especiais, etc.

No processo de Avaliação Logística, é importante o levantamento prévio dos recursos logísticos da região vulnerável, a fim de tornar mais rápido no momento da 
crise, o conhecimento dos recursos logísticos ainda disponíveis e os afetados, bem como as necessidades prementes da região. Para tal são definidas as seguintes entidades, que apresentam alguns relacionamentos entre si:

- Áreas de apoio: relaciona áreas de possível utilização para abrigos, atendimento a feridos, armazéns ou mesmo base de operação dentro da região atingida;

- Hospitais: relaciona os hospitais (incluindo a capacidade de atendimento e recursos) próximos ou internos à região de vulnerabilidade;

- Veículos/Equipamentos: relaciona os veículos (helicópteros, caminhões, etc) e equipamentos (retro-escavadeira, caminhão pipa, etc) de possível utilização na operação e sua disponibilidade, através de atributos como "contato", "órgão de origem", etc.;

- Rotas: relaciona as principais rotas na região de vulnerabilidade facilitando o mapeamento das disponíveis no pós-catástrofe;

- Fornecedores_Suprimentos: relaciona os suprimentos necessários (cobertores, barracas, vacinas, etc) e é definida pelos atributos como fornecedor e descrição, entre outros;

- Terceiros: relaciona empresas e serviços necessários no pós-catástrofe (construção civil, transportes, etc.);

\subsection{Protótipo do Banco de Dados de apoio a operações de resposta a catástrofes}

O modelo conceitual apresentado trata de um banco de dados direcionado ao uso desmembrado por regiões e cidades, as bases de dados a serem cadastradas neste compreendem pequena quantidade de dados, não demandando, portanto, o uso de ferramentas de grande porte. Assim, para implementação deste protótipo foi utilizado o Microsoft Access 2010 que possui a capacidade de tamanho de arquivo do banco de dados de dois gigabytes, e possibilidade de criação de tabelas de até 255 registros e é de fácil utilização. O Microsoft Access 2010 conta com funcionalidades que proporcionam fácil criação e manutenção de uma base de dados, atuando como gerenciador de banco de dados e sendo ao mesmo tempo uma ferramenta acessível a usuários não especializados. Desta forma, o protótipo apresentado foi criado já com a possibilidade de utilização via Web (também uma 
funcionalidade do Microsoft Access 2010), bastando para tal apenas ser disponibilizado em um servidor de acesso. A Figuras 1, 2 e 3 apresentam telas do protótipo implementado, onde figuram um formulário de entrada de dados e relatórios possíveis a partir da estrutura de dados criada e uma tabela, de forma a exemplificar a utilização do Modelo Conceitual desenvolvido. Na Figura 1, o relatório Dados dos Profissionais apresenta uma listagem dos profissionais cadastrados no sistema, ordenados por nome, exibindo todas as informações existentes no cadastro. Na Figura 2, o relatório Lista de Profissionais exibe uma listagem dos contatos dos profissionais cadastrados, ordenada por sobrenome e pode ser gerada por área de atuação. A Figura 3 apresenta uma visão dos registros da tabela Fornecedores de Suprimentos.

Figura 2 - Relatório do protótipo que exibe todos os dados dos profissionais Cadastrados incipal 15 DadosdosProfissionais

\section{Dados dos Profissionais}

\begin{tabular}{llll}
\hline Nome do Contato & Maria Garcia & Email & mariagarciabl@gmail.com \\
Área de Atuação & $\begin{array}{l}\text { Engenharia de } \\
\text { Transportes }\end{array}$ & Órgão de Origem & $\begin{array}{l}\text { Instituto Federal de Educação do } \\
\text { Sudeste de Minas Gerais }\end{array}$ \\
Sub-Área de Atuação & Sistemas de Informação & &
\end{tabular}

Figura 3 - Relatório do protótipo que lista os profissionais cadastrados

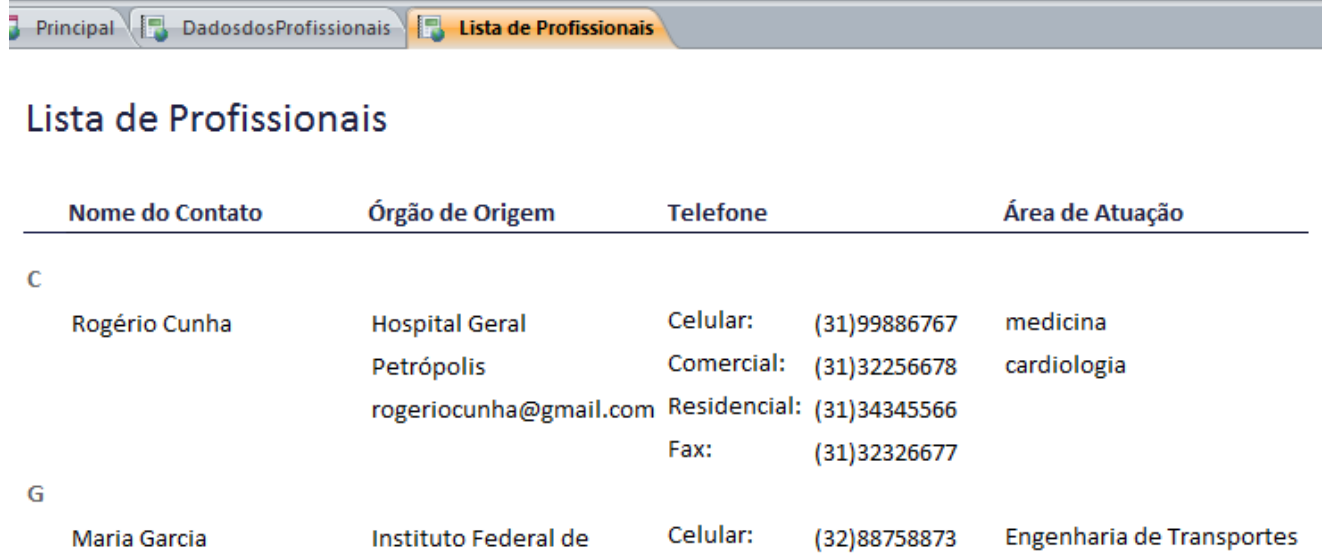

Figura 4 - Vista da tabela de Fornecedores de Suprimentos

\begin{tabular}{|c|c|c|c|c|c|c|c|c|c|c|}
\hline \multicolumn{4}{|c|}{ Logística Humanitária } & \multicolumn{7}{|c|}{ Ferramentas de Tabela } \\
\hline ternos & \multicolumn{3}{|c|}{ Ferramentas de Banco de Dados } & Campos & Tabela & & & & & \\
\hline \multicolumn{6}{|c|}{ Contatos $\mathrm{Ro}$} & \multicolumn{5}{|c|}{ Fornecedores de Suprimentos -7 Areas de Apoio } \\
\hline \multirow[t]{4}{*}{ digo } & $\cdot$ & Fornecedor - & Tipo de Forr & ecimento - & Cidade & Forn - & Descrição & Nome do Responsável & Contatos do Responsável . & Endereçc \\
\hline & 1 & Bretas & Alimentos & & Juiz de $\mathrm{F}$ & Fora & Cereais e Laticínios & José da Silva & (32)88778899 & R. Florest \\
\hline & 2 & Biomed & Medicament & & Juiz de $\mathrm{F}$ & Fora & Medicamentos, vaci & Maria José Cruz & (32) 88889192 & Av. Luz In \\
\hline & 3 & Quiral Químič & Medicament & & Juiz de $\mathrm{F}$ & Fora & Vacinas e materiais I & Jorge Alberto Ferreira & (32) 91262727 & BR-040 - - \\
\hline
\end{tabular}




\section{CONCLUSÃO}

Quando realizada de forma adequada e num rápido período de tempo, a organização da ajuda às vítimas, em seus diversos aspectos, reduz o sofrimento da população atingida. Desta forma, desenvolveu-se o framework proposto, evidenciando-se ações e atividades consideradas essenciais ao sucesso das operações de resposta a desastres naturais, de acordo com a importância percebida nas pesquisas e das intersecções existentes entre os diversos manuais. Este framework pode ter grande aplicação no Brasil, que não dispõe de muitos trabalhos focados na prática deste tipo de operação.

O objetivo do modelo proposto consiste em auxiliar e agilizar a estruturação de uma operação de resposta a desastres naturais no Brasil, tendo em vista a complexidade em se organizar este tipo de ação devido à dificuldade de obtenção de informações neste cenário e à diversidade de envolvidos e das atividades que precisam ser realizadas e gerenciadas no período pós-catástrofe. Considera-se que o framework proposto, por sua simplicidade e objetividade, pode auxiliar na tomada de decisões e implantação de processos nestas operações.

Além disto, o Modelo Conceitual de Banco de Dados proposto para a preparação da operação de resposta, já poderia ser implantado em regiões onde há recorrência de desastres, tornando-se um instrumento importante, tanto para as ações propostas no framework como no apoio ao processo decisório com base em informações de qualidade, cadastradas previamente e que vão assegurar agilidade nas decisões. O conhecimento prévio das condições dos hospitais da região, por exemplo, pode assegurar a correta escolha do encaminhamento das vítimas. É ainda grande a utilidade deste no processo de mobilização de diversos recursos (profissionais, equipamentos, veículos, prestadores de serviço) nas diversas ações da operação de resposta, sendo seu custo de utilização mínimo em relação aos benefícios que proporciona e amplamente aconselhável seu emprego em regiões com alta vulnerabilidade.

É importante ressaltar que os resultados deste trabalho além de apoiarem a atuação na logística humanitária, pode ainda ser a base para o desenvolvimento de estudos na área de gestão e produção que visem otimizar as ações propostas. 


\section{REFERÊNCIAS}

ALTAY, N., PRASAD, S. e SOUDERPANDIAN, J. Strategic planning for international disaster relief logistics: implications for research and practice. Richmond, Robins School of Business, University of Richmond. 31 p. 2006.

BALCIK, B. BEANIB, B.M. and SMILOWITZ.K.R. Last mile distribution in humanitarian relief. Journal of Intelligent Transportation Systems, v. 12, n. 2. p. 51-63, 2008.

http://dx.doi.org/10.1080/15472450802023329

BANDEIRA, R. A. M., CAMPOS, V. B. G. e BANDEIRA, A.P.F. Uma visão da logística de atendimento à população atingida por desastre natural. [Online]. XXV Congresso De Pesquisa e Ensino em Tranporte - Anpet, Belo Horizonte. 2011. Disponível em: http:// aquarius.ime.eb.br / webde2/prof/vania/pubs.htm [Capturado em 18 de março de 2012].

CASTRO, A. L. C. e CALHEIROS, L. B. Manual de medicina de desastres. Volume I. Brasília, Ministério da Integração Nacional, Secretaria Nacional de Defesa Civil. 2007. 91 p.

FEMA PUBLICATIONS WAREHOUSE. National response framework. U.S. Department of Homeland Security, Washington, DC. 2008. 90 p.

GAD-EL-HAK, M. Large-scale disasters: prediction, control, and mitigation. Ed. Ilustrada: Editora Cambridge University Press. 2008. 576 p.

GUNHA-SAPIR, D.; HOYOIS, P. e BELOW, R. Annual disaster statistical review 2012: The numbers and trends. Centre for Research on the Epidemiology of Disasters. Brussels: CRED; 2013. Available at: <http://cred.be/sites/default/files/ADSR_2012.pdf>. Accessed in november 13, 2013.

INTERNATIONAL COMMITTEE OF THE RED CROSS - IFRC [Online]. Guidelines for assessment in emergencies. International Federation of Red Cross, Geneva, Switzerland. 2008. 124 p. Disponível em: www.ifrc.org [Capturado em 12 novembro de 2011].

KOVACS, G. e SPENS, K. Humanitarian logistics in disaster relief operations. International Journal of Physical Distribution \& Logistics Management, v. 37, n. 2, p. 99-114, 2007. http://dx.doi.org/10.1108/09600030710734820

LOG CLUSTER ORG. [Online]. Logistics operational guide. Disponível em: http://www.logcluster. org/. [Capturado em 12 novembro de 2011].

MINISTRY OF CIVIL DEFENCE \& EMERGENCY MANAGEMENT. The guide to the national civil defence emergency management plan. 2006. PO Box 5010, Wellington, New Zealand. Revisado em 2009.

NATARAJARATHINAM, M.; CAPAR, I. e NARAYANAN, A. Managing supply chains in times of crisis: a review of literature and insights. International Journal of Physical Distribution and Logistics Management, v. 39, n. 7, p. 535-573, 2009.

http://dx.doi.org/10.1108/09600030910996251

OLIVEIRA, M. Manual gerenciamento de desastres: sistema de comando em operações. Florianópolis, Universidade Federal de Santa Catarina, Centro Universitário de Estudos e Pesquisas sobre Desastres. 2009. 74 p. 
SCHULZ, S.F. Disaster relief logistics: benefits of and impediments to cooperation between humanitarian organizations. Ed. Ilustrada: Editora Haupt Verlag AG. 2009. 269 p.

THOMAS, A. Humanitarian logistics: enabling disaster response. Fritz Institute. 2003. 15 p.

THOMAS, A., KOPCZAK, L. R. From logistics to supply chain management: the path forward in the humanitarian sector. Fritz Institute. 2005. 15 p.

UNICEF . Emergency field handbook: a guide for Unicef staff. New York, NY 10017 EUA, UNICEF Division of Communication. 2005. 417 p.

UNITED Nations World Food Programme - WFP. Emergency Field Operations Pocketbook. Rome, Italy, Office of Humanitarian Affairs Operations Department, World Food Programme. 2002. 344 p.

VAN WASSENHOVE, L. Humanitarian aid logistics: supply chain management in high gear. Journal of the Operational Research Society. 57, 475-498, 2006.

http://dx.doi.org/10.1057/palgrave.jors.2602125

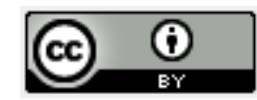

Artigo recebido em 17/06/2014 e aceito para publicação em 04/05/2015

DOI: http://dx.doi.org/ 10.14488/1676-1901.v15i2.1813 